\title{
Investigation of Optimal Prostate Biopsy Schemes for Chinese Patients with Different Clinical Characteristics
}

\author{
Ming-Kun Chen Yun Luo Hao Zhang Ming-Hua Lu Jun Pang Xin Gao \\ Department of Urology, The 3rd Affiliated Hospital of Sun Yat-Sen University, Guangzhou, PR China
}

\section{Key Words}

Prostate biopsy $\cdot$ Chinese men $\cdot$ Prostate cancer - Optimal scheme

\begin{abstract}
Purpose: To investigate the optimal schemes of prostate biopsy according to prostate volume (PV), age and transrectal ultrasound (TRUS) status in Chinese men. Methods: 923 consecutive patients who underwent initial TRUS-guided systematic 12-core prostate biopsy (12PBx) were enrolled in this study. The 12PBx was obtained by overlapping of conventional sextant, lateral base, mid-gland of peripheral zone and apex. Each sample was individually marked and inked before fixation. Patients were divided into 8 subgroups on the basis of independent risk factors investigated using logistic regression model. Subsequently, 12PBx was defined as self-control for the analysis of biopsy schemes (6-, 8- and $10 \mathrm{PBx}$ ) on individual core basis. The prostate cancer detection rates (CDRs) of 6-, 8-, 10- and 12PBx were compared for each individual subgroup. Results: The 12PBx detected 253 (27.4\%) cases of prostate cancer (PCa), of which 67.2, 47.1 and $61.3 \%$ were located in the base, mid-gland and apex, respectively. Multivariate analysis indicated that age, TRUS status and PV were independent risk factors for PCa detection. CDR increased with increasing biopsy cores. However, for patients with age $\geq 65$ years, positive TRUS and $P V<38.5 \mathrm{~cm}^{3}$,
\end{abstract}

CDR of $8 \mathrm{PBx}$ (30.6\%) was similar to $10 \mathrm{PBx}$ (32.2\%) and $12 \mathrm{PBx}$ (32.2\%); for patients with age $\geq 65$ years, negative TRUS and $\mathrm{PV}<38.5 \mathrm{~cm}^{3}$ or ones with age $\geq 65$ years, positive TRUS and $\mathrm{PV} \geq 38.5 \mathrm{~cm}^{3}, 10 \mathrm{PBx}$ was as effective as $12 \mathrm{PBx}$ in detecting PCa (27.8, 27.5 vs. 28.9, 29.3\%, respectively). Conclusion: Age, TRUS status and PV were independent risk factors for PCa detection. Traditional sextant biopsy is not recommended. $8-, 10-$, or $12 \mathrm{PBx}$ as an individual biopsy scheme might be adopted according to these risk factors for Chinese patients.

Copyright $\odot 2012$ S. Karger AG, Basel

\section{Introduction}

Prostate cancer $(\mathrm{PCa})$ is one of the most common malignant carcinomas among men in the world [1]. Transrectal ultrasound (TRUS)-guided needle biopsy is the standard diagnostic strategy before clinical decision-making such as radical prostatectomy or androgen deprivation [2]. Since the systematic sextant prostate biopsy protocol introduced by Hodge et al. [3], several clinicians have demonstrated that traditional standard sextant biopsies significantly underestimated the detection rates of $\mathrm{PCa}$

M.K.C. and Y.L. contributed equally to this article.

\section{KARGER}

Fax +4161306 1234

E-Mail karger@karger.ch

www.karger.com (c) 2012 S. Karger AG, Basel

0042-1138/12/0894-0425\$38.00/0

Accessible online at:

www.karger.com/uin
Xin Gao

Department of Urology

The 3rd Affiliated Hospital of Sun Yat-Sen University

Guangzhou, Guangdong 510630 (PR China)

Tel. +86 208525 2052, E-Mail xin.gao.zsu@gmail.com 
Fig. 1. Prostate biopsy protocol and core distribution. a Protocol of TRUS-guided prostate biopsy. b $12 \mathrm{PBx}$ biopsy scheme: traditional sextant biopsy (No. 1-6), 2 lateral base of peripheral zone cores (No. 7 and 8), 2 apex cores (No. 9 and 10) and 2 mid-gland cores of the peripheral zone (No. 11 and 12).

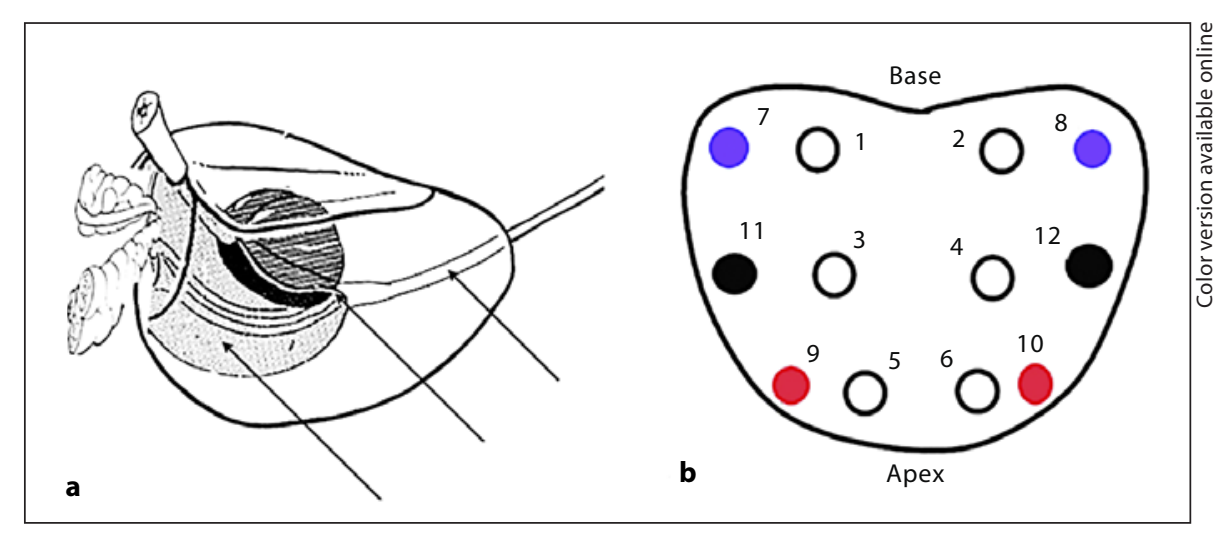

and strongly recommended an extensive scheme that ranged from 8 to 24 samples [4-7]. However, several studies reported that the increasing number of biopsy cores had not significantly improved the cancer detection rate (CDR), especially in the Asian population [8-11]. Abd et al. [11] indicate that there is no significant difference in the CDR between the 12-core biopsy scheme and the 8 -core biopsy protocol in the American. Despite the dramatically increasing incidence of $\mathrm{PCa}$ in China $[1,12]$, very limited data or experiences from Chinese patients in this field have been reported. Therefore, it is of great value to determine a prostate biopsy scheme for Chinese patients.

The most efficient number and location of prostate biopsies remain controversial. The necessity whether to rely on the same protocol or to modify it according to the different clinical parameters is still unclear. Recently, predictive models involved in serum PSA, digital rectal examination (DRE) status, age, and prostate volume (PV) have been designed to evaluate the risk of suffering from PCa $[10,13,14]$. According to these models, the investigators are allowed to quantify the combined contribution of several risk factors and provide a predicted probability of PCa. Scattoni et al. [6] have reported that the most advantageous biopsy schemes involved cores and sites that permitted the detection of $95 \%$ PCa by a 24 -core prostate biopsy (24PBx) approach according to different preoperative clinical characteristics for Europeans. Dai et al. [15] have introduced an individualized biopsy strategy for Chinese men with different PSA levels, but DRE status, PV, age and TRUS findings have not been investigated. As yet, risk factors for PCa detection and individual biopsy scheme based on these clinical risk factors have not been mapped up.
In the present study, we identify independent risk factors for $\mathrm{PCa}$ detection using logistic regression model, and compare the CDRs of 6-, 8-, 10-, and 12PBx in patients with different clinical parameters. The purpose of this study is to optimize individualized biopsy scheme for Chinese men according to their clinical characteristics.

\section{Patients and Methods}

From October 2000 to October 2011, the records of 1,862 patients undergoing TRUS-guided prostate biopsy in the Third Hospital of Sun Yat-Sen University were reviewed and analyzed. This study was conducted in accordance with the guidelines of the human ethics review committee at our Institute.

\section{Study Design and Case Selection}

A total of 1,862 patients underwent detailed clinical examinations, including serum levels of PSA, DRE, and TRUS. The indications to perform an initial biopsy were PSA $<10 \mathrm{ng} / \mathrm{ml}$ and/or fPSA $\%<0.1$ plus abnormal DRE and/or TRUS findings or PSA $>10 \mathrm{ng} / \mathrm{ml}$ regardless of DRE and TRUS status $[6,16]$.

Patients aged 50-80 years having PSA levels between 4 and $20 \mathrm{ng} / \mathrm{ml}$ and $12 \mathrm{PBx}$ were enrolled in our series. Patients who previously underwent needle biopsy or TURP and had a history of acute urinary retention, an indwelling catheter or evidence of acute urinary infection within 3 months before PSA measurement were excluded. Finally, a total of 923 men were included in the study. Demographic, clinical and laboratory data were collected including age, body mass index (BMI), pre-biopsy serum PSA, PV, DRE status and TRUS finding and medication profiles. $\mathrm{PV}$ was calculated using the standard ellipsoid formula.

\section{Biopsy Protocol}

A TRUS-guided needle biopsy was conducted by two experienced urologists (M.K.C. and L.Y.). We used an 18-gauge needle powered by a spring-loaded automatic biopsy gun offering 22-mm puncturing length. 12PBx overlapping the standard sextant biopsy, lateral base, mid-gland and apex of peripheral zone was performed for all the enrolled patients (fig. 1a). Subsequent- 
Table 1. Baseline characteristics of 923 patients

\begin{tabular}{lc}
\hline Variable & Patients \\
\hline Age, years & $65 \pm 7.9$ \\
$<65$ & $450(48.8 \%)$ \\
$\geq 65$ & $473(51.2 \%)$ \\
PSA, ng/ml & $10.6 \pm 7.8$ \\
$4-10$ & $438(47.5 \%)$ \\
$10.1-20$ & $485(52.5 \%)$ \\
Free PSA, $\%$ & $0.21 \pm 0.08$ \\
$<0.21$ & $491(53.2 \%)$ \\
$\geq 0.21$ & $432(46.8 \%)$ \\
Prostate volume, ml & $38.5 \pm 24.1$ \\
$<38.5$ & $468(50.7 \%)$ \\
$\geq 38.5$ & $455(49.3 \%)$ \\
PSA density & $0.27 \pm 0.11$ \\
$<0.27$ & $447(48.4 \%)$ \\
$\geq 0.27$ & $476(51.6 \%)$ \\
Body mass index, kg/m ${ }^{2}$ & $22.1 \pm 5.3$ \\
$\quad \leq 22.1$ & $449(48.6 \%)$ \\
$>22.1$ & $474(51.4 \%)$ \\
DRE status & $256(27.7 \%)$ \\
PCa-positive & $147(57.4 \%)$ \\
PCa-negative & $109(42.6 \%)$ \\
Suspicious lesions on TRUS & $299(32.4 \%)$ \\
PCa-positive & $161(53.8 \%)$ \\
PCa-negative & $138(46.2 \%)$ \\
Biopsy finding & \\
PCa & $253(27.4 \%)$ \\
HGPIN & $168(18.3 \%)$ \\
ASAP & $33(3.5 \%)$ \\
BPH & $469(50.8 \%)$ \\
\hline & \\
&
\end{tabular}

HGPIN = High-grade prostatic intraepithelial neoplasia; ASAP = atypical small acinar proliferation of prostate; $\mathrm{BPH}=$ benign prostatic hyperplasia.

ly, each biopsy specimen was individually marked (fig. 1b) before fixation by using $10 \%$ formalin, and the pathological diagnosis was recorded. In patients who had the indication of lesions suspicious of PCa (hypoechoic areas or asymmetries predominantly in the peripheral zone) on TRUS, a target biopsy core was performed. This core took place of the nearest core of the $12 \mathrm{PBx}$ procedure. Each block was reviewed by an experienced uropathologist using contemporary diagnostic criteria for highgrade prostatic intraepithelial neoplasia, lesion suspicious but not diagnostic of adenocarcinoma (atypical small acinar proliferation of prostate), and PCa.

\section{Statistical Analysis}

For analysis, 6 core schedules included No. 1-6, which was accordance with conventional sextant biopsy; sextant biopsy cores plus two lateral bases of the peripheral zone (No. 7 and 8) was considered as 8 -core regimen $(8 \mathrm{PBx}) ; 8 \mathrm{PBx}$ plus two apex cores (No. 9 and 10) was defined as 10 -core protocol (10PBx), and $12 \mathrm{PBx}$
Table 2. Clinical and pathological characteristics of $253 \mathrm{PCa}$ patients

\begin{tabular}{lc}
\hline Variable & Patients \\
\hline Age, years & $65 \pm 13.6$ \\
PSA, ng/ml & $11.6 \pm 6.7$ \\
$\quad 4-10$ & $118(46.6 \%)$ \\
$10.1-20$ & $135(53.4 \%)$ \\
Prostate volume, ml & $37.3 \pm 21.5$ \\
PSA density & $0.29 \pm 0.11$ \\
DRE positive & $147(58.1 \%)$ \\
Suspicious lesions on TRUS & $161(63.6 \%)$ \\
Tumor location & \\
$\quad$ Base & $170(67.2 \%)$ \\
Mid-gland & $119(47.1 \%)$ \\
Apex & $155(61.3 \%)$ \\
Gleason score & \\
2-6 & $153(60.5 \%)$ \\
7 & $57(22.5 \%)$ \\
$8-10$ & $43(17.0 \%)$ \\
Positive cores (mean) & $1-12(3.2 \%)$ \\
T classification & \\
T1c & $76(30.1 \%)$ \\
T2a & $36(14.2 \%)$ \\
T2b & $60(23.7 \%)$ \\
T2c & $57(22.5 \%)$ \\
T3 & $17(6.7 \%)$ \\
T4 & $7(2.8 \%)$ \\
\hline
\end{tabular}

included sextant biopsy, mid-gland, lateral base and apex of the peripheral zone (fig. 1b). We calculated the CDRs of 6-, 8-, 10- and $12 \mathrm{PBx}$ based on each individual core. CDRs of 6-, 8-, 10- and $12 \mathrm{PBx}$ were compared using the $\chi^{2}$ test. Continuous variables are presented as mean \pm standard deviation. One-way analysis of variance was used to compare means. For multivariate analyses, logistic regression was introduced on the basis of the outcomes of univariate analysis. Independent risk factors of suffering $\mathrm{PCa}$ were identified using logistic regression model. Patients were grouped according to risk factors of suffering PCa. The level of significance was determined to be a p value $<0.05$. The SPSS software package, version 16.0 (Chicago, III., USA), was used for all statistical analysis.

\section{Results}

\section{Patient Characteristics and Biopsy Findings}

In total, 923 patients were included in our study. The details of baseline characteristics of these patients are summarized in table 1 . Briefly, the DRE findings were suspicious $\mathrm{PCa}$ in $27.7 \%$ of the 923 patients [ 256 men including 147 (57.4\%) patients with pathological malignant 
Table 3. Univariate and multivariate analysis of risk factors for PCa detection

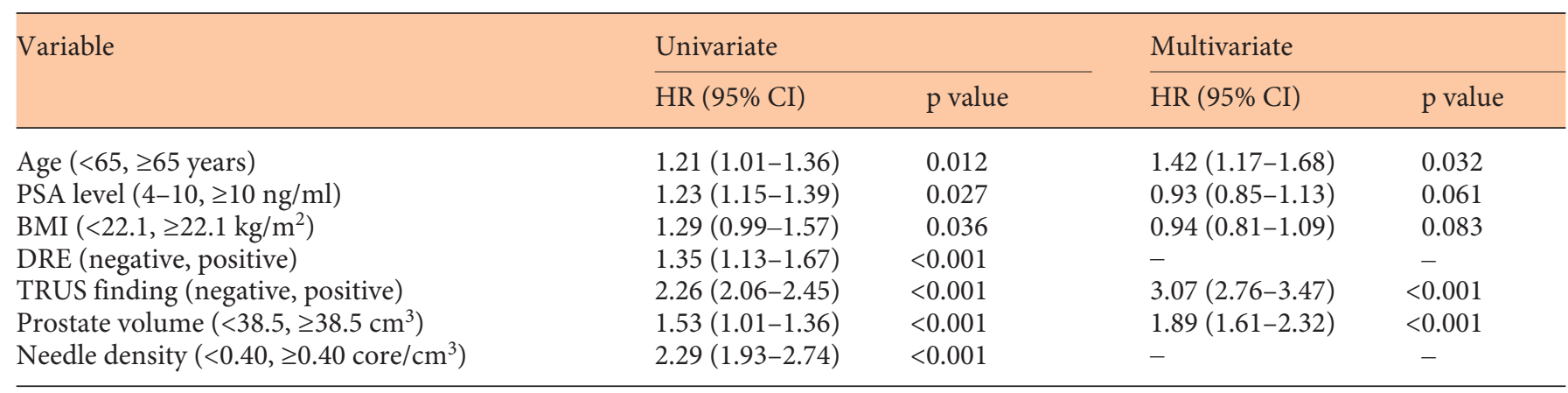

findings and 109 (42.6\%) patients with non-malignant lesions]. Suspicious lesions were detected on TRUS in 299 (32.4\%) patients, including 161 (53.8\%) cases with $\mathrm{PCa}$ and $138(46.2 \%)$ patients with non-PCa. At the initial biopsy, 253 (27.4\%) PCa, 168 (18.3\%) PIN, 33 (3.5\%) ASAH and $469(50.8 \%)$ benign lesions were detected.

Of the 253 men with positive PCa focus, $76,153,17$ and 7 patients were diagnosed as clinical stage T1c, T2, T3, T4 cancer, respectively. 153 cases had Gleason score $<7$, and 100 men with Gleason score $\geq 7$. Abnormal DRE findings were detected in 147 patients (58.1\%). 161 patients (63.6\%) had suspicious PCa lesions on TRUS. The mean positive cores were 3.2 (range 1-12). The details of clinical and pathological information of the 253 patients with PCa are shown in table 2. Moreover, 170 (67.2\%), 155 (61.3\%) and 119 (47.1\%) patients were noticed to have positive PCa focus in the base, apex and middle gland, respectively. We set the cancer-positive rate of $12 \mathrm{PBx}$ at $100 \%$. Traditional sextant biopsy program detected $62.8 \%$ of $\mathrm{PCa}$ focus. $75.5 \%$ of PCa could be detected if additional two lateral base cores were added on the basis of sextant biopsy strategy (8PBx). And $90.5 \%$ of $\mathrm{PCa}$ could be detected by $10 \mathrm{PBx}$ approach. Only additional $8.5 \%$ of PCa could be detected if two mid-gland of peripheral zone cores were performed. The rate of positive PCa for each core of the $12 \mathrm{PBx}$ in 253 patients with positive focus is shown in figure 2.

\section{Univariate and Multivariate Models for $\mathrm{PCa}$}

Detection

We found that PSA level (4-10, 10-20 ng/ml), age ( $<65$, $\geq 65$ years), DRE and TRUS findings (positive or negative), PV $\left(<38.5, \geq 38.5 \mathrm{~cm}^{3}\right)$ and needle biopsy density $(<0.4$ or $\geq 0.40)$ indicated different $\mathrm{PCa}$ detection rate in the univariate model as shown in table 3 (all p values $<0.05)$. TRUS was more accurate than DRE on detecting

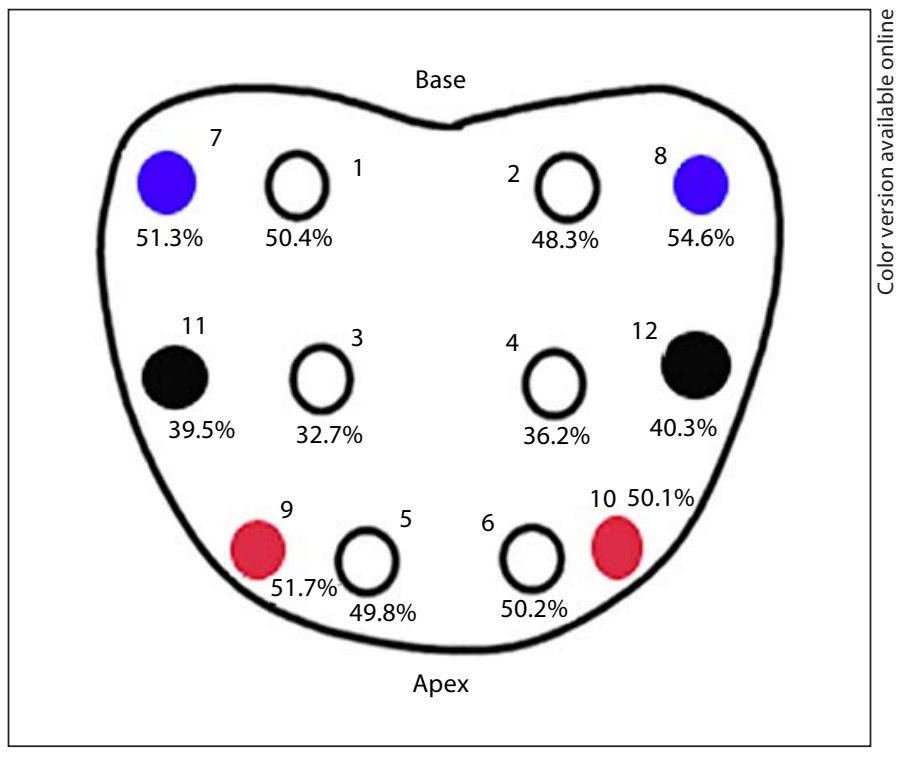

Fig. 2. Positive percentage on an individual-core basis of $12 \mathrm{PBx}$ in 253 PCa patients.

abnormal prostate lesions. All the patients in this study received $12 \mathrm{PBx}$, needle density depending on the PV. Thus, age, PSA level, TRUS and PV were included in the multivariate model. We found that age (HR: $1.42,95 \% \mathrm{CI}$ : 1.17-1.68, $\mathrm{p}=0.032$ ), TRUS lesions (HR: 3.07, 95\% CI: 2.76-3.47, p < 0.001) and PV (HR: 1.89, 95\% CI: 1.61-2.32, $\mathrm{p}<0.001)$ were independent risk factors for predicting $\mathrm{PCa}$ on the 12PBx procedure in multivariate logistic regression model, while serum PSA level (HR: 0.93, 95\% CI: $0.85-1.13, \mathrm{p}=0.061$ ) and BMI (HR: 0.94, 95\% CI: 0.81$1.09, \mathrm{p}=0.083)$ were not an independent risk factor for PCa detection (table 3 ). 
Fig. 3. A total of 923 cases were divided into eight subgroups according to age, $\mathrm{PV}$ and TRUS finding.

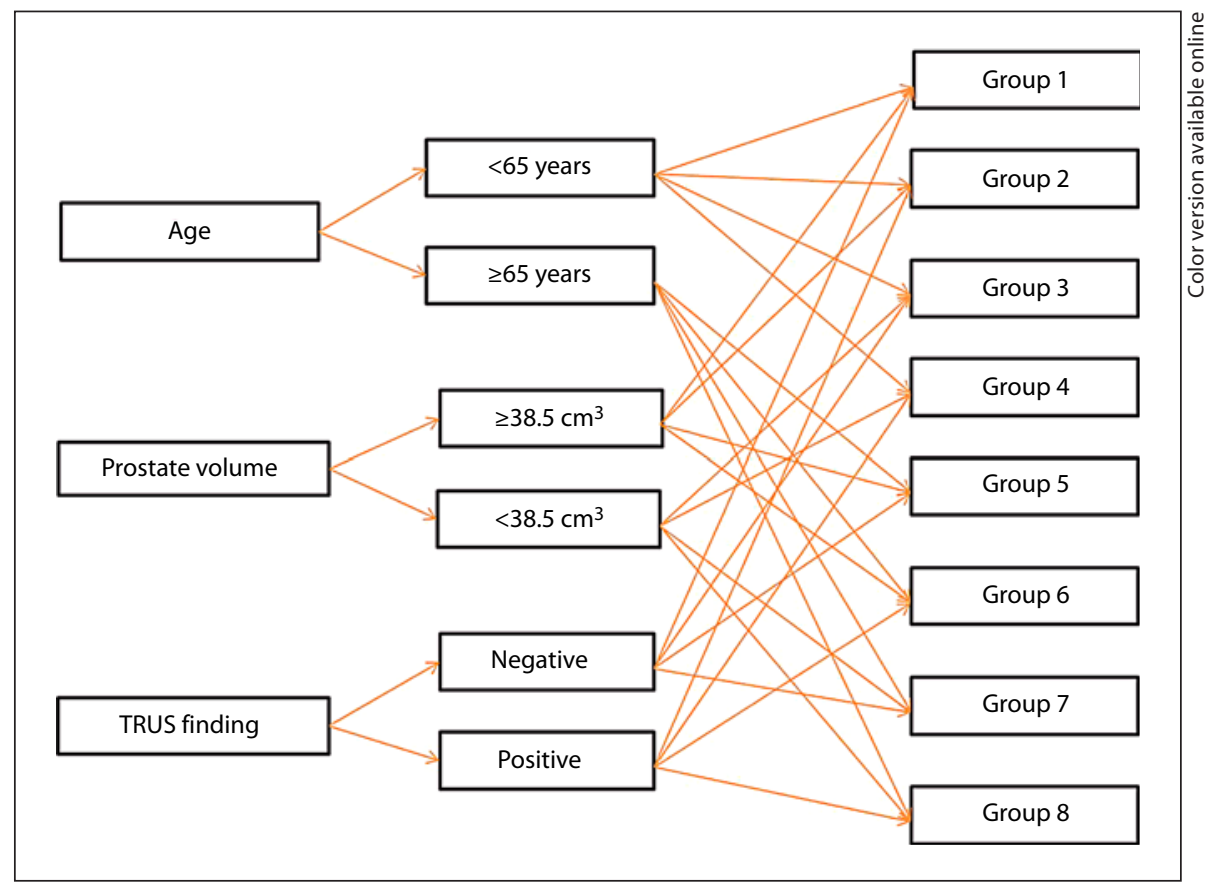

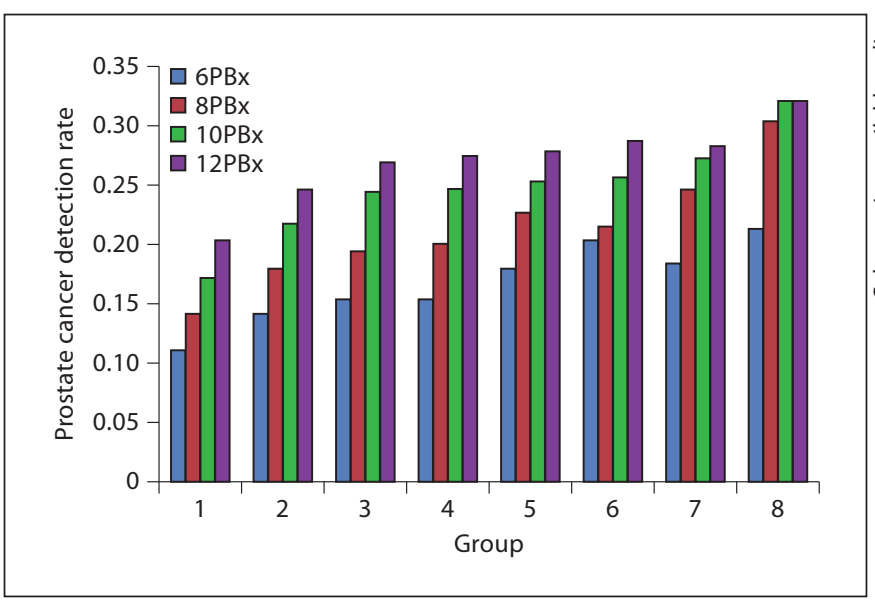

Fig. 4. Comparison of CDRs of 6-, 8-, 10- and 12PBx for 8 subgroups of patients on the basis of different clinical characteristics.

\section{Optimal Biopsy Schemes for Different Clinical}

\section{Characteristics}

In the multivariate model, we found that age, TRUS findings and PV were independent risk factors for detecting PCa. Subsequently, we divided this group of patients into eight subgroups based on the risk factors for $\mathrm{PCa}$ detection involved in age, TRUS findings and PV (fig. 3). PCa detection rate of sextant, 8 cores, 10 and 12 cores were compared for each individual subgroup of patients. In patients older than 65 years, negative TRUS and with $\mathrm{PV}<38.5 \mathrm{~cm}^{3}$, PCa detection rate of 10PBx was $27.8 \%$. It was significantly higher than $20.6 \%$ of $6 \mathrm{PBx}$ and $22.8 \%$ of $8 \mathrm{PBx}(\mathrm{p}=0.012$ and 0.037 , respectively), but nearly the same as $28.9 \%$ of $12 \mathrm{PBx}$ protocol $(\mathrm{p}=0.12)$. Similar result was found among patients older than 65 years, positive TRUS and with PV $\geq 38.5 \mathrm{~cm}^{3}$. Patients who were older than 65 years, positive TRUS and with $\mathrm{PV}<38.5 \mathrm{ml}, 6-, 8-, 10-$ and $12 \mathrm{PBx}$ detected 25.5, 30.6, 32.2 and $32.2 \%$ of $\mathrm{PCa}$, respectively. PCa detection rate of $8 \mathrm{PBx}$ was significantly higher than $6 \mathrm{PBx}(\mathrm{p}=0.031)$, similar to $10 \mathrm{PBx}$ and $12 \mathrm{PBx}(\mathrm{p}=0.112$ and 0.112 , respectively). PCa detection rate increased with increasing biopsy cores for patients in groups 1-5. PCa detection rates of $12 \mathrm{PBx}$ were $20.4,24.7,27.0,27.5$ and $28.1 \%$, respectively (fig. 4).

\section{Discussion}

Our institute has been outfitted with TRUS equipment in 2000, and since then TRUS-guided prostate biopsy was routinely performed in 1,862 patients. In this study, we focused on 923 cases with serum PSA level from 4 to $20 \mathrm{ng} / \mathrm{ml}$ to decrease the possible biases in the analyses. 
The overall CDR was $27.4 \%$ using a $12 \mathrm{PBx}$ procedure, which was consistent with a previous study in another Chinese center. Dai et al. [15] demonstrated a CDR of $22.2 \%$ by a $10 \mathrm{PBx}$ in the subgroup of patients with a PSA level of 4-20 ng/ml. Gao et al. [17] detected $33 \mathrm{PCa}$ in 113 (29.2\%) patients with a PSA level lower than $20 \mathrm{ng} / \mathrm{ml}$. However, this rate is lower than the results from North American and European countries [11]. Abd et al. [11] reported an overall positive biopsy rate of $49.2 \%$ using a 12core biopsy strategy. The explanation is that the PSA level in that study ranged from 0.1 to $5,000 \mathrm{ng} / \mathrm{ml}$ with a mean value of $14.7 \mathrm{ng} / \mathrm{ml}$ compared with a PSA level of $4-20 \mathrm{ng} / \mathrm{ml}$, with $4-10 \mathrm{ng} / \mathrm{ml}$ in $47.5 \%$ of patients of this study; in addition, abnormal DRE was observed in $51.5 \%$ of enrolled patients, while only $27.4 \%$ of 923 cases had suspicious lesion on DRE in our series. Moreover, higher CDR (35-50\%) caused by more biopsy cores was also demonstrated in several studies. Outcomes from Guichard showed that CDR using 18 biopsy samples and 21 samples were 41.5 , and $42.5 \%$, respectively.

Many clinical studies demonstrated that the proportion of detecting PCa varied with different clinical characteristics of patients including age, serum PSA level, DRE status, TRUS finding, PV biopsy cores and needle density [10]. Clinical judgment was biased for all of these clinical characteristics, and limitations occurred. Consequently, some statistical models have been developed to circumvent these biases. Nowadays, logistic-based nomograms, risk groupings, artificial neural networks, probability tables, and classification and regression tree analyses were available $[10,18]$. Many risk factors such as family history, age, PSA level, BMI, DRE status, TRUS and PV were identified using these predictive models. In this study, we adopted a logistic regression model to identify risk factors for detecting PCa, and age, TRUS finding and $\mathrm{PV}$ were identified as independent risk factors for $\mathrm{PCa}$ detection.

It has been reported that the ability to detect PCa was related to both the biopsy site and the number of biopsy cores taken $[13,19]$. Since the introduction of conventional sextant biopsy approach by Hodge et al. [3], several authors have demonstrated that extensive biopsy schemes involved the far lateral peripheral zone, and the apex was crucial to optimize the CDR [20-23]. Meng et al. [21] indicated that the apex and the base of the peripheral gland are the sites where PCa is most likely located and where the biopsies should be directed. Our study showed that $67.2,47.1$ and $61.3 \%$ of positive cores were localized in the base, middle gland and apex of the peripheral gland, respectively, which was consistent with the results of previous reports. In the analysis of positive core distribution in our patients, only $62.8 \%$ of positive cores were observed in the traditional sextant biopsy program in 253 patients with $\mathrm{PCa}$ when we set the cancer-positive rate of $12 \mathrm{PBx}$ at $100 \%$. In contrast, 75.5 and $90.5 \%$ of positive cores were detected using $8 \mathrm{PBx}$ and $10 \mathrm{PBx}$, respectively. These outcomes confirmed that additional biopsy cores in the lateral base and apex can significantly increase CDR, conventional sextant was insufficient for effectively detecting PCa, and more cores localized in far lateral base of peripheral zone and apex should be added. As shown in figure $1 \mathrm{~b}$, two lateral base cores, two apex cores and two mid-gland cores of the peripheral zone were added to conventional sextant biopsy protocol, respectively, which was defined as 8-core, 10-core and 12-core biopsy protocol.

Scattoni et al. [20] found that the most advantageous biopsy schemes including cores and location varied from patients with different clinical characteristics involved in age, PV, DRE status, and demonstrated that $10 \mathrm{PBx}$ could detect $95 \% \mathrm{PCa}$ detected by $24 \mathrm{PBx}$ in patients with positive DRE lesion. Neill et al. [24] recently reported that a protocol of medial sextant and targeted biopsy revealed about $95 \%$ of PCa that would be detected with a 10-core biopsy protocol. Luciani et al. [25] showed that in patients presenting with abnormal DRE findings, a transperineal 6-core biopsy yielded a detection rate similar to that of a 12-core biopsy. These studies indicated that the biopsy protocol should be modified and individual biopsy schemes involving cores and sites should be adopted on the basis of patients' clinical characteristics. Dai et al. [15] reported an individualized biopsy program according to different PSA levels for Chinese patients, and revealed that $10 \mathrm{PBx}$ overlapping standard sextant, two far lateral base cores and two mid-gland of peripheral zone was the best individual scheme for patients with PSA level $<20 \mathrm{ng} / \mathrm{ml}$. However, age, TRUS finding and PV were not taken into account. In this study, 8 subgroups of patients were systematically identified according to age, TRUS finding and PV. The optimal biopsy scheme for each subgroup of patients was investigated. In patients older than 65 years, negative TRUS and with PV $<38.5 \mathrm{~cm}^{3}$ or patients older than 65 years, positive TRUS and with $P V \geq 38.5 \mathrm{~cm}^{3}$, $10 \mathrm{PBx}$ was as effective as $12 \mathrm{PBx}$ in $\mathrm{PCa}$ detection. Moreover, 8 cores are enough for patients older than 65 years, positive TRUS and PV $<38.5 \mathrm{~cm}^{3}$. The explanation could be that older age of patients, smaller volume of the prostate, higher core density and suspicious lesion-targeted biopsy resulted in an increased CDR. Thus, the 
decreased core biopsy program can be applied to these subgroups of patients, and the personalized biopsy scheme should be considered rather than a systemic biopsy schedule for every patient. The outcomes of the present study indicated that traditional sextant biopsy was not suggested for Chinese patients, but 8-, 10- or $12 \mathrm{PBx}$ can be individually applied based on age, TRUS status and PV.

\section{Conclusions}

In the present study, age, TRUS finding and PV were identified as independent risk factors for PCa detection. Our investigation evidenced that an individual biopsy protocol involving cores and sites can be recommended for Chinese patients based on their own clinical characteristic patterns. The traditional sextant prostate biopsy approach was not recommended for all Chinese patients. Patients older than 65 years, negative TRUS and with PV $<38.5 \mathrm{~cm}^{3}$ or patients older than 65 years, pos- itive TRUS and with $\mathrm{PV}>38.5 \mathrm{~cm}^{3}$ should receive $10 \mathrm{PBx}$ as an individual biopsy protocol. A personalized $8 \mathrm{PBx}$ program can be adopted for patients older than 65 years, positive TRUS and with PV $<38.5 \mathrm{~cm}^{3}$. However, it is better to conduct a 12PBx procedure for cases with other patterns of age, TRUS status and PV.

\section{Acknowledgements}

This study was supported by the Program of 5010 of Sun YatSen University (2007028), Chinese National Natural Science Foundation (30973011), Key Project of Chinese Ministry of Health (10120165), Chinese National Hi-Tech Research and Development Program (2007AA021906), and Chinese National Natural Science Foundation (30901496).

\section{Disclosure Statement}

The authors declare no conflicts of interest.

\section{References}

1 Jemal A, Siegel R, Ward E, Hao Y, Xu J, Thun MJ: Cancer statistics, 2009. CA Cancer J Clin 2009;59:225-249.

${ }_{2}$ Ta S, Klausner AP, Savage SJ, Unger P, BarChama N: Male infertility due to a benign prostatic polyp. J Urol 2000;164:1659-1660.

- 3 Hodge KK, McNeal JE, Stamey TA: Ultrasound guided transrectal core biopsies of the palpably abnormal prostate. J Urol 1989;142: 66-70.

-4 Guichard G, Larre S, Gallina A, Lazar A, Faucon H, Chemama S, Allory Y, Patard JJ, Vordos D, Hoznek A, Yiou R, Salomon L, Abbou CC, de la Taille A: Extended 21-sample needle biopsy protocol for diagnosis of prostate cancer in 1000 consecutive patients. Eur Urol 2007;52:430-435.

5 Kawakami S, Okuno T, Yonese J, Igari T, Arai G, Fujii Y, Kageyama Y, Fukui I, Kihara K: Optimal sampling sites for repeat prostate biopsy: a recursive partitioning analysis of three-dimensional 26-core systematic biopsy. Eur Urol 2007;51:675-682, discussion 682-673.

-6 Scattoni V, Raber M, Abdollah F, Roscigno M, Deho F, Angiolilli D, Maccagnano C, Gallina A, Capitanio U, Freschi M, Doglioni C, Rigatti P, Montorsi F: Biopsy schemes with the fewest cores for detecting $95 \%$ of the prostate cancers detected by a 24-core biopsy. Eur Urol 2010;57:1-8.
Ploussard G, Xylinas E, Salomon L, Allory Y, Vordos D, Hoznek A, Abbou CC, de la Taille A: The role of biopsy core number in selecting prostate cancer patients for active surveillance. Eur Urol 2009;56:891-898.

8 Matsumoto K, Satoh T, Egawa S, Shimura S, Kuwao S, Baba S: Efficacy and morbidity of transrectal ultrasound-guided 12-core biopsy for detection of prostate cancer in Japanese men. Int J Urol 2005;12:353-360.

9 Kim JW LH, Hong SJ, Chung BH: Can a 12 core prostate biopsy increase the detection rate of prostate cancer versus 6 core? A prospective randomized study in Korea. Yonsei Med J 2004;45:671-675.

10 Suzuki H, Komiya A, Kamiya N, Imamoto T, Kawamura K, Miura J, Suzuki N, Nakatsu H, Hata A, Ichikawa T: Development of a nomogram to predict probability of positive initial prostate biopsy among Japanese patients. Urology 2006;67:131-136.

- 11 Abd TT, Goodman M, Hall J, Ritenour CW, Petros JA, Marshall FF, Issa MM: Comparison of 12 -core versus 8 -core prostate biopsy: multivariate analysis of large series of US veterans. Urology 2011;77:541-547.

$12 \mathrm{Gu}$ F: Epidemiological survey of benign prostatic hyperplasia and prostatic cancer in China. Chin Med J (Engl) 2000;113:299-302.
3 Shim HB, Park HK, Lee SE, Ku JH: Optimal site and number of biopsy cores according to prostate volume prostate cancer detection in Korea. Urology 2007;69:902-906.

- 14 Kawakami S, Numao N, Okubo Y, Koga F, Yamamoto S, Saito K, Fujii Y, Yonese J, Masuda H, Kihara K, Fukui I: Development, validation, and head-to-head comparison of logistic regression-based nomograms and artificial neural network models predicting prostate cancer on initial extended biopsy. Eur Urol 2008;54:601-611.

15 Dai B, Ye DW, Kong YY, Shen YJ, Wang BH Individualized prostate biopsy strategy for Chinese patients with different prostate-specific antigen levels. Asian J Androl 2008; 10 : 325-331.

16 Zaytoun OM, Kattan MW, Moussa AS, Li J, Yu C, Jones JS: Development of improved nomogram for prediction of outcome of initial prostate biopsy using readily available clinical information. Urology 2011;78:392398.

17 Gao HW, Li YL, Wu S, Wang YS, Zhang HF, Pan YZ, Zhang L, Tateno H, Sato I, Kuwahara M, Zhao XJ: Mass screening of prostate cancer in a Chinese population: The relationship between pathological features of prostate cancer and serum prostate specific antigen. Asian J Androl 2005;7:159-163. 
18 Stephan C, Cammann H, Semjonow A, Diamandis EP, Wymenga LF, Lein M, Sinha P, Loening SA, Jung K: Multicenter evaluation of an artificial neural network to increase the prostate cancer detection rate and reduce unnecessary biopsies. Clin Chem 2002;48: 1279-1287.

19 Haas GP, Delongchamps NB, Jones RF, Chandan V, Serio AM, Vickers AJ, Jumbelic M, Threatte G, Korets R, Lilja H, de la Roza G: Needle biopsies on autopsy prostates: sensitivity of cancer detection based on true prevalence. J Natl Cancer Inst 2007;99:14841489.
20 Scattoni V, Zlotta A, Montironi R, Schulman C, Rigatti P, Montorsi F: Extended and saturation prostatic biopsy in the diagnosis and characterisation of prostate cancer: a critical analysis of the literature. Eur Urol 2007;52: 1309-1322.

-21 Meng MV, Franks JH, Presti JC Jr, Shinohara $\mathrm{K}$ : The utility of apical anterior horn biopsies in prostate cancer detection. Urol Oncol 2003;21:361-365.

-22 Singh H, Canto EI, Shariat SF, Kadmon D, Miles BJ, Wheeler TM, Slawin KM: Improved detection of clinically significant, curable prostate cancer with systematic 12core biopsy. J Urol 2004;171:1089-1092.

23 Iremashvili V, Pelaez L, Jorda M, Manoharan $\mathrm{M}$, Arianayagam M, Rosenberg DL, Soloway MS: Prostate sampling by 12 -core biopsy: comparison of the biopsy results with tumor location in prostatectomy specimens. Urology 2012;79:37-42.
24 Neill MG, Toi A, Lockwood GA, Evans A, Tammsalu L, Fleshner NE: Systematic lateral prostate biopsy - are the benefits worth the costs? J Urol 2008;179:1321-1326.

25 Luciani LG, De Giorgi G, Valotto C, Zanin M, Bierti S, Zattoni F: Role of transperineal six-core prostate biopsy in patients with prostate-specific antigen level greater than $10 \mathrm{ng} / \mathrm{ml}$ and abnormal digital rectal examination findings. Urology 2006;67:555-558. 